\title{
Multiple Food Additives Enhance Human Chronic Diseases
}

\author{
Aaron Lerner ${ }^{1,2 *}$, Torsten Matthias ${ }^{2}$ \\ ${ }^{1}$ B Rappaport School of Medicine, Technion-Israel Institute of Technology, Haifa, Israel \\ ${ }^{2}$ AESKU.KIPP Institute, Wendelsheim, Germany
}

Received: April 19, 2016; Accepted: June 22, 2016; Published: June 25, 2016

*Corresponding author: Aaron Lerner, Aesku.Kipp Institute, Mikroforum ring 2, Wendelsheim 55234, Germany, Tel: +49-6734-9622-1010; Fax: +49-6734-9622-2222; E-mail: aaronlerner1948@gmail.com

Keywords: Food additive; Chronic disease; P-80, Intestinal permeability; Microbiota; Mucus; Intestine

\section{Introduction}

In the current issue of SOJ Microbiology \& Infectious Diseases a frequently used emulsifying food additive: Polysorbate-80 (P80) was shown to impact mouse microbiota, promote intestinal inflammation, obesity and liver dysfunction [1]. Epidemiological data provide strong evidence of a steady rise in autoimmune and allergic diseases throughout westernized societies over the last decades [2]. This trend parallels the recent pandemic of the metabolic syndrome and other lifestyle related human disorders $[3,4]$. The present editorial will expand on additional food additives, used heavily in the processed food industries, which join P-80 as environmental inducers of chronic diseases.

\section{Food Additive Consumption is Increasing}

Living in western countries has a strong impact on nutritional habits collectively termed the "Western diet" including high fat, trans fatty acids, cholesterol, proteins, sugars, salt intake, as well as frequent consumption of processed and "fast food" [3]. The net fold \% increases/year of the 7 industrial food additives sales and consumption over the last 4-6 decades was summarized recently [5-7]. As reported, in an increasing order, emulsifiers, salt, industrial food enzymes and nanoparticles are sold and consumed the most.

\section{Food Additives Drive Human Microbiome towards Dysbiosis}

The human gastrointestinal tract exhibits one of the highest densities of microorganisms on earth. Several studies have analyzed the microbiota in the large intestine showing that nutrition affects the intestine luminal bacteria, thus impacting the host's well-being. The question arises, if food additives impact the intestinal ecosystem? The absolute answer is positive. Glucose [8], salt [9], organic solvents [10], emulsifiers [1,11,12], gluten $[13,14]$, microbial transglutaminase $[5,6]$, and nanoparticles [15] impact the intestinal ecosystem, affecting the equilibrium between the microbiota and the dysbiota.

\section{Food Additives affect the Intestinal Mucus}

A defective mucosal barrier may result in an increased intestinal permeability. Thus, promoting the exposition to luminal content and triggers an immunological response that promotes intestinal inflammation. In this regard, food additives like emulsifiers $[1,11,16]$, nanoparticles [17] and various Western diet components [18] were described as breaching the intestinal mucus layer integrity.

\section{Food Additives Breach Tight Junction (TJ) Integrity}

Over recent decades, a significant increase in the incidence of chronic immune mediated diseases in industrialized countries has led to the postulation that diet is a potential environmental risk factor for such disorders. Only a single layer of epithelial cells separates the luminal contents from effector immune cells in the lamina propria and the internal milieu of the body. Breaching this single layer of epithelium can lead to pathological exposure of the highly immunoreactive sub epithelium to the large number of foreign antigens in the lumen. In fact, breached TJ integrity is observed in multiple infectious, autoimmune and allergic diseases. It was suggested that commonly used industrial food additives play a major part in increased intestinal permeability. The seven food additives mentioned above [5-7], abrogate human epithelial barrier function and increase intestinal permeability through the opened TJ, enhancing the entry of foreign immunogenic antigens and activation of the sub-epithelial immune cascade [1,5-7].

\section{Summary}

Multiple food additives might be detrimental to human health, by inducing disequilibrium between the microbiota and dysbiota and by compromising the intestinal barrier mechanisms. Figure 1 summarizes the mechanism of increased intestinal permeability induced by food additives used in the processed food industries. $\mathrm{P}-80$ is only one of them [1]. Its emulsifying capacity join multiple additional pathways were intestinal protective mechanisms are disrupted. Further studies on the effects of industrial processed food additives on the intestinal ecosystem events, mucus integrity and intestinal permeability will impact on the food industry additive policy, food products labeling, consumer awareness, regulatory authorities and public health implementation. 


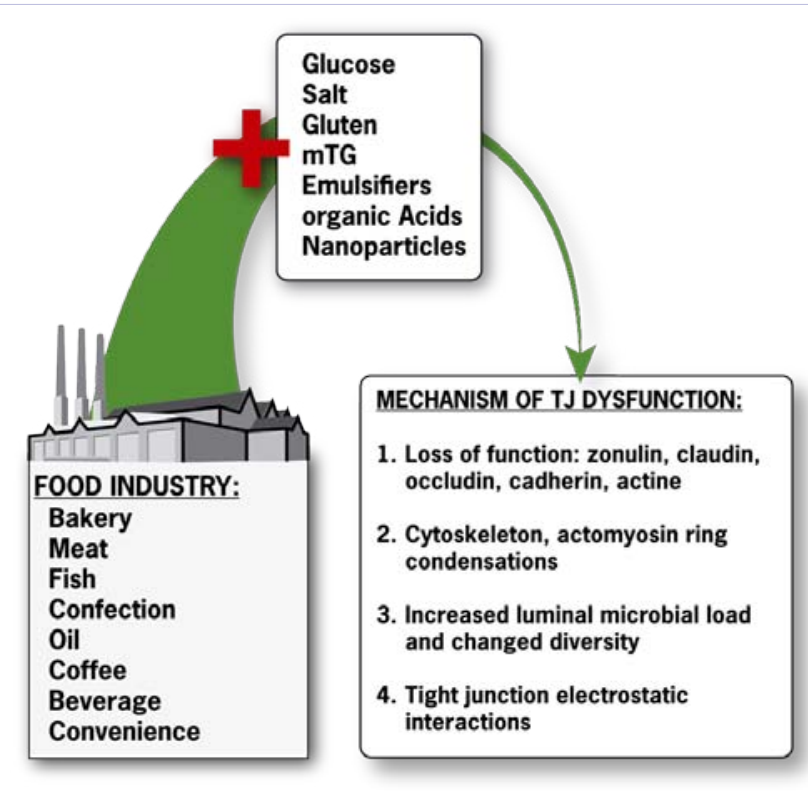

Figure 1: Mechanism of increased intestinal permeability induced by food additives used in the processed food industries.

\section{References}

1. Singh RK, Wheildon N, Ishikawa S. Food Additive P-80 Impacts Mouse Gut Microbiota Promoting Intestinal Inflammation, Obesity and Liver Dysfunction. SOJ Microbiol Infect Dis. 2016;4(1):1-10.

2. Bach JF. The effect of infections on susceptibility to autoimmune and allergic diseases. N Engl J Med. 2002;347(12):911-20.

3. Manzel A, Muller DN, Hafler DA, Erdman SE, Linker RA, Kleinewietfeld M. Role of "Western diet" in inflammatory autoimmune diseases. Curr Allergy Asthma Rep. 2014;14(1):404. doi: 10.1007/s11882-0130404-6.

4. Han TS, Lean ME. A clinical perspective of obesity, metabolic syndrome and cardiovascular disease. JRSM Cardiovasc Dis. 2016;5:2048004016633371. doi: 10.1177/2048004016633371.

5. Lerner A, Matthias T. Changes in intestinal tight junction permeability associated with industrial food additives explain the rising incidence of autoimmune disease. Autoimmun Rev. 2015;14(6):479-89. doi: 10.1016/j.autrev.2015.01.009.

6. Lerner A, Matthias T. Possible association between celiac disease and bacterial transglutaminase in food processing: a hypothesis. Nutr Rev. 2015;73(8):544-52. doi: 10.1093/nutrit/nuv011.
7. Lerner A, Trinder $\mathrm{P}$, Matthias T. Breaching intestinal tight junction permeability associated with industrial food additives might explain the surge in autoimmune disease incidence. In: From Autoantibody Research to Standardized Diagnostic Assays in the Management of Human Diseases. Volume 10th, Eds: K Conrad, Chan EKL, Andrade LEC, Steiner G, Pruijn GJM, Y Shoenfeld. 12th symposium on autoantibodies, 23-26.9.15, Dresden, Germany. Page 10-16, Pabst Science Publishers, Lengerich, Germany, e-pub.

8. Utzschneider KM, Kratz M, Damman CJ, Hullarg M. Mechanisms Linking the Gut Microbiome and Glucose Metabolism. J Clin Endocrinol Metab. 2016;101(4):1445-54. doi: 10.1210/jc.2015-4251.

9. Jose PA, Yang Z, Zeng C, Felder RA. The importance of the gastrorenal axis in the control of body sodium homeostasis. Exp Physiol. 2016;101(4):465-70. doi: 10.1113/EP085286.

10. He X, Slupsky CM. Metabolic fingerprint of dimethyl sulfone (DMSO2) in microbial-mammalian co-metabolism. J Proteome Res. 2014;13(12):5281-92. doi: 10.1021/pr500629t.

11. Chassaing B, Koren O, Goodrich JK, Poole AC, Srinivasan S, Ley RE, et al. Dietary emulsifiers impact the mouse gut microbiota promoting colitis and metabolic syndrome. Nature. 2015;519(7541):92-6. doi: $10.1038 /$ nature14232.

12. Cani PD. Metabolism: Dietary emulsifiers--sweepers of the gut lining? Nat Rev Endocrinol. 2015;11(6):319-20. doi: 10.1038/ nrendo.2015.59.

13. Sanz Y. Microbiome and Gluten. Ann Nutr Metab. 2015;67 Suppl 2: 2841. doi: $10.1159 / 000440991$.

14. Leonard MM, Fasano A. The microbiome as a possible target to prevent celiac disease. Expert Rev Gastroenterol Hepatol. 2016;10(5):555-6. doi: 10.1586/17474124.2016.1166954.

15. Fröhlich EE, Fröhlich E. Cytotoxicity of Nanoparticles Contained in Food on Intestinal Cells and the Gut Microbiota. Int J Mol Sci. 2016;17(4). pii: E509. doi: 10.3390/ijms17040509.

16. Csáki KF. Synthetic surfactant food additives can cause intestinal barrier dysfunction. Med Hypotheses. 2011;76(5):676-81. doi: 10.1016/j.mehy.2011.01.030.

17. Wilcox MD, Van Rooij LK, Chater PI, Pereira de Sousa I, Pearson JP. The effect of nanoparticle permeation on the bulk rheological properties of mucus from the small intestine. Eur J Pharm Biopharm. 2015;96:4847. doi: 10.1016/j.ejpb.2015.02.029.

18. Martinez-Medina M, Denizot J, Dreux N, Robin F, Billard E, Bonnet R, et al. Western diet induces dysbiosis with increased $E$ coli in CEABAC10 mice, alters host barrier function favouring AIEC colonisation. Gut. 2014;63(1):116-24. doi: 10.1136/gutjnl-2012-304119. 